\section{Cytokine storm barrier}

\section{By Michael J. Haas, Senior Writer}

Researchers from the U.S. and Brazil have found that the protein slit homolog 2 can prevent tissue damage and death caused by the cytokine storm during bacterial sepsis or severe viral infections. ${ }^{1}$ Navigen Pharmaceuticals has in-licensed the findings and thinks the protein's enhancement of vascular stability could be more effective at treating hypercytokinemia than strategies that target individual cytokines.

As part of the body's normal inflammatory response to bacterial and viral infections, immune cells secrete proinflammatory cytokines that disrupt intercellular interactions between cellular adhesion molecules that form the vascular barrier. This allows immune cells to permeate the barrier and reach infected tissues.

After moving across the vascular barrier, the immune cells ordinarily close the cytokineinduced gaps. But during sepsis and other severe infections, the inflammatory response can spiral out of control, resulting in hypercytokinemia. The flood of proinflammatory cytokines causes excessive vascular permeability, which can lead to vascular leakage, tissue edema, organ failure and death.

Therapeutics on the market or in develop-

\section{"Anything that works on the vascular component of inflammation has a good chance of successfully treating sepsis and hypercytokinemia." - Charles Serhan, Harvard Medical School}

bacterial endotoxin-induced lung injury, peritoneal microbial sepsis following intestinal puncture and $\mathrm{H} 5 \mathrm{~N} 1$ influenza infection. In all three models, the SLIT2 fragment increased vascular stability, reduced signs of vascular damage in organs and increased survival compared with no treatment.

$\mathrm{Li}$ is a professor of medicine, oncological sciences and human genetics at the University of Utah. The team also included researchers from the Utah State University, the Oswaldo Cruz Foundation, the Howard Hughes Medical Institute and the Stanford University School of Medicine.

Data were published in Science Translational Medicine.

\section{Weathering the storm}

Targeting vascular permeability could be a better strategy to treat the cytokine storm than inhibiting cytokines directly, which often has undesirable immunosuppressive effects.

"People used to think of the endothelium as silent tubes" that were passive participants in inflammatory processes, said Charles Serhan, professor of anesthesiology at Harvard Medical School. "But now we know it is where the first step of inflammation occurs as cytokines move across the endothelial barrier. Anything that works on the vascular component of inflammation has a good chance of successfully treating sepsis and hypercytokinemia."

Serhan is also director of the Center of Experimental Therapeutics and Reperfusion Injury at Brigham and Women's Hospital and a cofounder of Resolvyx Pharmaceuticals Inc.

"Helping vasculature weather the storm by strengthening endothelial cell interactions and thereby decreasing vascular permeability would be a novel way to provide therapeutic benefit during the acute inflammatory phase of sepsis and severe viral infections," said Resolvyx CSO Philip Vickers.

Serhan said the acute lung damage model used by Li's team was especially relevant to the hospital setting-where sepsis is treatedbecause it mimicked lung damage and consequent sepsis caused by mechanical ventilation.

Resolvyx is developing a class of endogenous molecules called resolvins to promote the resolution of various inflammatory conditions. A Serhan-led research group discovered resolvins in 2000. Last year, his team showed that in a mouse model of sepsis, resolvin D2 (RvD2) reduced levels of proinflammatory cytokines and promoted bacterial clearance and survival compared with vehicle. ${ }^{3}$

"The mechanisms of action of SLIT2 and resolvin D2 might be different, in that SLIT2 is solely targeting the vascular leak" whereas RvD2 is a master regulator of all cytokine activity, Serhan said. "But both are ultimately dialing down the cytokines to control the cytokine storm and reduce vascular leakage."

Vickers noted that SLIT2's mechanism of action, which is distinct from that of resolvins, steroids and antimicrobials, "could complement 
these approaches and might work well in combination therapy."

$\mathrm{Li}$ agreed that SLIT2 or its mimetics would most likely be used as adjuvants to treat sepsis. He added that SLIT2-based therapies could have broad utility in sepsis and hypercytokinemia because "using them does not require knowing the precise pathogen responsible for the infection."

\section{Windows of opportunity}

According to Navigen CBO Brandi Simpson, the company is developing Slit2N-the SLIT2

fragment used by the Li team-as well as smaller Slit $2 \mathrm{~N}$ fragments to treat conditions characterized by cytokine-induced vascular leakage. "Navigen has also identified small molecules that affect the same downstream signaling pathway and appear to prevent vascular leakage," she said.

Vickers said it will be important to see whether SLIT2 or a mimetic has a therapeutic effect after the onset of sepsis. He said a key limitation of the study by Li's team was giving the SLIT2 fragment prior to inducing disease in the mice. "In the clinical setting, where patients present with sepsis symptoms, the initial cytokine storm will likely have passed," he noted.

Li agreed that "how early one can give-or has to give-SLIT2 will impact the likelihood of a successful outcome" in preventing cytokine storm-induced tissue damage and death. "Our guess is that there is a window between infection, cytokine storm and complete vascular collapse" in which SLIT2 treatment would be effective.

But Li noted that the size of that window is currently uncertain because it will depend on when a patient begins to experience symptoms such as fever, malaise and muscle pain, which signal the onset of hypercytokinemia.

Vickers also said the normal biological roles of SLIT2, ROBO4 and related proteins have not been fully characterized in vivo. "Thus, it is uncertain whether modulation of interactions between these proteinsor the consequent effects on general endothelial barrier function-may result in unanticipated biological consequences."

As an example, he cited a 2008 review by U.K. researchers at the University of Birmingham College of Medical and Dental Sciences that described roles for SLIT and ROBO family proteins in tumor angiogenesis. ${ }^{4}$

Li said it was too early to hypothesize about SLIT2's side-effect profile. But even if SLIT2 were found to have side effects, those still might be acceptable "given that the outcomes of sepsis and hypercytokinemia are so bleak," he said.

Li added: "We would imagine that in the case of acute sepsis, influenza or biodefense emergency you would give SLIT2 for a limited time," which would minimize any side effects associated with long-term treatment.

The findings reported in Science Translational Medicine are covered by a patent issued to the University of Utah and have been licensed to Navigen, of which Li is a cofounder.

In addition to Slit $2 \mathrm{~N}$ and its fragments, Simpson said the company is exploring potential therapeutic applications of agents that target SLIT2 and ROBO4 signaling to treat sepsis, acute respiratory distress syndrome (ARDS), pulmonary fibrosis, rheumatoid arthritis (RA) and other indications.

Haas, M.J. SciBX 3(13); doi:10.1038/scibx.2010.395

Published online April 1, 2010

\section{REFERENCES}

1. London, N. et al. Sci. Transl. Med.; published online March 17, 2010; doi:10.1126/scitranslmed.3000678

Contact: Dean Y. Li, The University of Utah, Salt Lake City, Utah e-mail: dean.li@hmbg.utah.edu

2. Jones, C. et al. Nat. Med. 14, 448-453 (2008)

3. Spite, M. et al. Nature 461, 1287-1291 (2009)

4. Legg, J. et al. Angiogenesis 11, 13-21 (2008)

COMPANIES AND INSTITUTIONS MENTIONED

Brigham and Women's Hospital, Boston, Mass.

Harvard Medical School, Boston, Mass.

Howard Hughes Medical Institute, Chevy Chase, Md.

Navigen Pharmaceuticals, Salt Lake City, Utah

Oswaldo Cruz Foundation, Rio de Janeiro, Brazil

Resolvyx Pharmaceuticals Inc., Bedford, Mass.

Stanford University School of Medicine, Stanford, Calif.

University of Birmingham College of Medical and Dental Sciences,

Birmingham, U.K.

The University of Utah, Salt Lake City, Utah

Utah State University, Logan, Utah 